\title{
Self-Reporting Materials: Protein-Mediated Visual Indication of Damage in a Bulk Polymer
}

\author{
Nico Bruns $\S^{\S a}$ and Douglas S. Clark \\ §SCS DSM Prize for best poster
}

\begin{abstract}
Damage self-reporting materials are able to indicate the presence of microscopic damaged regions by easy to detect signals, such as fluorescence. Therefore, these smart materials can reduce the risk of catastrophic failure of load-bearing components, e.g. in aerospace and construction applications. We highlight here our proof-of-concept paper and we present some additional data, which shows that proteins can be used as mechanophores in solid polymeric materials. Macroscopic mechanical forces were transferred from the polymer to the embedded proteins. The biomolecules act as molecular strain sensor, giving the material the desired selfreporting property. Poly(ethylene glycol) and poly(acrylamide) (PAAm) networks were doped with small amounts of thermsosome (THS), a protein cage from the family of chaperonins, that encapsulated a pair of fluorescent proteins. THS acts as a scaffold which brings the two fluorescent proteins into distance suitable for fluorescence resonance energy transfer (FRET). Moreover, THS can be distorted by mechanic forces so that the distance between the fluorescent proteins changes, leading to a change in FRET efficiency. Using the brittle PAAm as a model system, we were able to visualize microcracks in the polymers by FRET microscopy and by fluorescence lifetime imaging. THS also stabilizes the encapsulated guest proteins against thermal denaturation, increasing their half-live at $70^{\circ} \mathrm{C}$ by a factor of 2.3 .
\end{abstract}

Keywords: Chaperonin · Damage detection $\cdot$ Hybrid material $\cdot$ Mechanophore $\cdot$ Polymer

\section{Introduction}

Polymeric materials are omnipresent in modern societies and find applications ranging from packaging, to biomedical materials, to lightweight construction and load-bearing materials. Their excellent and tunable mechanical properties and the possibility to mold them into any desired shape are just two reasons for their widespread use. Most polymers are static materials that do not change their properties in response to a change in their environment or an external stimulus. However, smart materials

\footnotetext{
${ }^{*}$ Correspondence: Dr. N. Bruns ${ }^{a}$

Tel.: +41612673832

Fax: + 41612673855

E-mail: nico.bruns@unibas.ch

aDepartment of Chemistry

University of Basel

Klingelbergstr. 80

$\mathrm{CH}-4056$ Basel

${ }^{b}$ Department of Chemical and Biomolecular

Engineering

University of California

Berkeley, CA 94720, USA
}

that possess responsive properties have emerged over the last decades, one type being polymeric materials that respond to a mechanical input. ${ }^{[1]}$ Self-repairing materials, in particular, have attracted widespread interest. ${ }^{[2-4]}$ These materials autonomously repair themselves after incurring damage, such as microcracks or even full fractures, thus prolonging the life of the material and reducing the risk of catastrophic material failure under load.

Complementary to self-repairing materials are materials that autonomously indicate to the user the presence or occurrence of damage by readily detectable signals, preferably before microdamage has spread and enlarged to hazardous macroscopic damages. Such systems would allow the user to replace or repair damaged components in due time, with the result of lowering the risk of accidents. Moreover, these systems could also serve the purpose of quality control at the start of a product's life-cycle, indicating that the product left the factory and reached the customer without any microdamage. We refer to these kinds of materials as 'self-reporting', in analogy to the term 'self-repairing'. However, only a few systems are known to possess this particular self- $X$ property.

A simple but effective method to generate self-reporting materials is to embed dye- filled microcapsules ${ }^{[5]}$ or hollow fibers ${ }^{[6-9]}$ into polymeric materials. Upon deformation, the capsules or fibers break, releasing their content into damage-induced microcavities, such as cracks and delaminated areas, giving rise to colored regions. This concept has often been implemented as an added feature to self-healing systems based on reagent-filled capsules or fibers. Another possibility to produce self-reporting materials is based on electrically or optically conductive fibers in a composite material, such fibers changing their conductive properties upon damage. ${ }^{[10]}$ The aforementioned methods are based on macro- or microscopic objects that are embedded in the polymer material. As such, they might alter the mechanical properties of the materials (for better or for worse) and, most importantly, they are not able to detect mechanical forces or damage on the molecular level. However, macroscopic damage is always preceded by events on the molecular level, e.g. the displacement of polymer chains, chain scission, or delamination at the interface between a carbon fiber and a polymer resin. Molecular force sensors, i.e. mechanochemically-sensitive reactive molecules, so called mechanophores, offer the possibility of detecting and visualizing damage or deformation through mechanically-induced chemical changes at the mo- 
lecular scale. ${ }^{[1]}$ Recently explored classes of mechanophores in polymeric materials include spiropyrans, which produce a color change upon stress-induced electrocyclic ring-opening, ${ }^{[11,12]}$ cyclobutane-containing polymers of tricinnamate, which undergo a cycloreversion reaction upon crack-formation accompanied by a change in the fluorescence emission spectra, ${ }^{[13]}$ and dyeaggregates whose external force-triggered dissociation can change the dye's photoluminiscent character. [14-19]

We are investigating a different class of mechanophores: proteins that can report mechanical forces by a change in their fluorescence properties. Using proteins as functional components in polymer-protein hybrid materials permits exploiting the multiverse of tunable properties of these biomolecules in technical applications. The proteins offered by Nature are intriguing functional nanodevices whose three-dimensional structures can be mechanically distorted. Some proteins are mechanophores on their own; for example, fluorescent proteins, which lose fluorescence upon mechanical unfolding. [20,21] However, a successful implementation of this property in a solid material has not been reported yet. Other proteins are known to have mechanically weak spots and planes, but do not themselves fluoresce. However, they can be engineered to display fluorophores.

We decided to take advantage of the mechanical properties and the scaffold structure of an archaeal chaperonin, the thermosome (THS) from Thermoplasma acidophilum. THS is a cage-like hexadecameric protein assembly, composed of two stacked, octameric rings that enclose two central cavities (Fig. 1a). ${ }^{[22]}$ Each cavity is large enough to accommodate a protein guest. Indeed, the natural function of chaperonins is to encapsulate (partially) unfolded proteins into their cavities in order to refold them. ${ }^{[23]}$ In its closed conformation, the THS resembles a hollow sphere approx. $16 \mathrm{~nm}$ in diameter, in which the rings join in an equatorial plane. This plane is known to be the mechanically weak spot of the chaperonins, as AFM experiments have revealed that one ring of the chaperonin GroEL is easily separated from the other with the tip of the cantilever, leaving the rings intact. ${ }^{[24]}$ The centers of the cavities of THS are approx. 5-8 nm apart, which is close to the Förster radius of pairs of fluorescent proteins in fluorescence resonance energy transfer (FRET). [25] Thus, THS can be used as a scaffold in which to place an enhanced cyan fluorescent protein (eCFP) and an enhanced yellow fluorescent protein (eYFP) at a distance most suitable to monitor distance changes via FRET (Fig. 1b). Here we will highlight our proof-ofconcept paper, in which we demonstrated

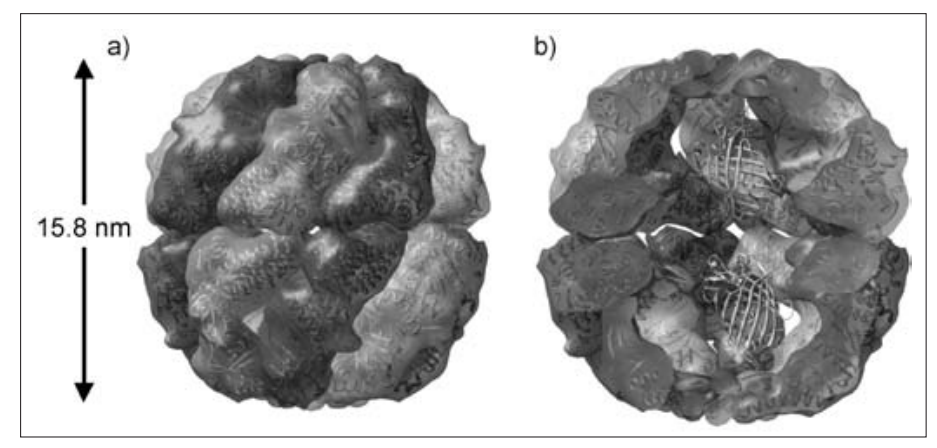

Fig. 1. a) Structure of THS in its closed conformation. Side view generated from the PDB entry $1 \mathrm{A6D} \cdot{ }^{[22]}$ b) Cut-away model of THS encapsulating a pair of fluorescent proteins. The model shows the size relation between the guests and the cavities of THS. Please note that the model does not represent the actual conformation in solution or in the polymers, as THS rests in an open conformation in the absence of ATP. ${ }^{[23]}$ However, no crystal structure of this conformation is available.

that this multi-protein complex is capable of reporting microcrack formation induced by uniaxial strain of a brittle, transparent polymer.[26] Moreover, we will present some additional data showing the response of the hybrid material to damage and investigate the THS-imposed temperature stabilization of the fluorescent proteins.

\section{Results and Discussion}

\section{Preparation of Thermosome-eCFP- eYFP Conjugate}

Chaperonins are protein cages that have large openings through which protein substrates can enter and leave the cavities. In the case of group II chaperonins, to which THS belongs, these pores are gated by built-in lids.[23] The lids are formed of helical protrusions in the apical domain of subunits which open and close in an irislike movement, powered by the hydrolysis of ATP. ${ }^{[27]}$ In the absence of ATP, the protein rests in a fully or partially open conformation, thus the cavities are accessible from solution. The challenges were to devise a method to coencapsulate two fluorescent proteins in the THS, to ensure that the guest proteins remain in the protein cage permanently, leaving the guest proteins in their native conformation in order to exhibit fluorescence.

A chemical conjugation strategy was chosen to entrap fully-folded fluorescent proteins in the THS. First, the THS was genetically engineered by three point-mutations to display cysteine residues within its cavity, but not on the outside surface. A heterobifunctional linker, maleimido trioxa-6-formyl benzamide (MTFB), was attached to these attachment points, which introduced aromatic aldehyde groups into the cavities. In parallel, eCFP and eYFP were modified with the complementary linker by reacting amine groups of surface-exposed lysines with succinimidyl 6-hydrazinonicotinate acetone hydrazone (SANH, also abbreviated S-HyNic). THS-fluorescent protein conjugates were formed upon simple mixing of buffered solutions of the three proteins and incubation at room temperature. These conjugates were then purified by size-exclusion chromatography, as proven by UV-Vis spectroscopy and gel-electrophoresis. The fluorescent proteins diffused into the THS's cavities, and the very efficient reaction of aromatic aldehydes with the hydrazines then formed bisaryl hydrazone bonds between the THS and the fluorescent proteins, effectively entrapping guests in the protein cage. The formation of the linker is observable by UV-Vis spectroscopy because the formed bond gives rise to an adsorption band with a maximum at 352 $\mathrm{nm}$. Thus, UV-Vis spectra were used to determine the average numbers of eCFP and eYFP per THS, as well as the number of linker-bonds formed. A typical spectrum revealed 1.6 hydrazone bonds per fluorescent protein, as well as 0.4 eCFP and 0.7 eYFP per THS. For FRET to occur, a pair of eCFP and eYFP has to be entrapped in the same THS. The population contained the desired species, which were verified by fluorescence emission spectroscopy. A solution of the conjugate was irradiated with UV light at wavelengths that excite the FRET donor, eCFP. The fluorescence emission spectra were corrected for spectral bleed-through and cross-talk, which resulted in the typical emission spectra of eYFP, the FRET acceptor. The intensitybased FRET measurements were supported by fluorescence lifetime imaging (FLIM) measurements. The mean fluorescence lifetime of the eCFP depends on its microenvironment. Most notably, energy transfer to a FRET acceptor decreases the fluorescence lifetime. Thus, a shorter mean lifetime indicates higher FRET efficiency. Moreover, FLIM enabled measurement of the inter-fluorophore distance to be $5.23 \pm$ 
$0.13 \mathrm{~nm}$, which nicely corresponds to the distance between the two cavities of THS.

Because the uptake of fluorescent proteins into THS is a statistical process, it is expected that mixed populations of singlyand doubly-filled protein cages as well as empty ones were obtained. This inefficiency in preparing FRET systems using THS as a scaffold was compensated by the ease of the method of preparing the conjugates, i.e. just mixing and waiting. Moreover, the analytical methods used, intensity-based FRET and FLIM, allow the extraction of FRET signal from the raw data, thus making a separation of the FRET-active THSeCFP-eYFP conjugates from the rest of the THS population unnecessary.

\section{THS Stabilizes a Protein Guest against Thermal Denaturation}

The role of THS in biology is to refold partially unfolded proteins. It prolongs the life of other proteins; e.g. when the organism is exposed to heat shock. We therefore examined whether the THS would have a positive effect on the thermal stability of the encapsulated protein guests. Fluorescent proteins are known to lose their fluorescence at temperatures higher than approx. $60{ }^{\circ} \mathrm{C}$ due to thermal denaturation. ${ }^{[28]}$ Indeed, we measured a rapid decay in fluorescence emission of eYFP in aqueous solution at $70{ }^{\circ} \mathrm{C}$ (Fig. 2). This decay could be described by first-order kinetics, giving a half-life of $87 \mathrm{~s}$. When the THS-eCFPeYFP conjugate was heated to $70{ }^{\circ} \mathrm{C}$, the fluorescence of the encapsulated eYFP still decayed, but with more than a 2-fold longer half life of 207 s. Thus, the chaperonin did stabilize its enclosed guest significantly.

\section{Synthesis of Polymer-Protein Hybrid Materials}

In order to covalently incorporate THS-eCFP-eYFP into polymers, the protein conjugates were first reacted with $\mathrm{N}$ succinimidyl acrylate to yield acrylamidemodified lysines on the protein's surface. These functional groups were then copolymerized with acrylic monomers by free radical polymerization in aqueous solution, followed by a drying step.

Copolymerization with the monomer $\alpha, \omega$-acrylate-terminated poly(ethylene glycol) yielded soft, flexible and turbid materials (PEG), whereas a mixture of acrylamide and the cross-linker $N, N^{\prime}$ methylene-bis-acrylamide produced hard, brittle, and transparent samples (PAAm). Both materials did fluoresce, due to the incorporated proteins, while control samples that did not contain any protein showed no fluorescence (Fig. 3). Relatively little protein was needed to obtain self-reporting materials. Typical samples contained only 0.1 or 0.2 wt $\%$ THS-eCFP-eYFP.

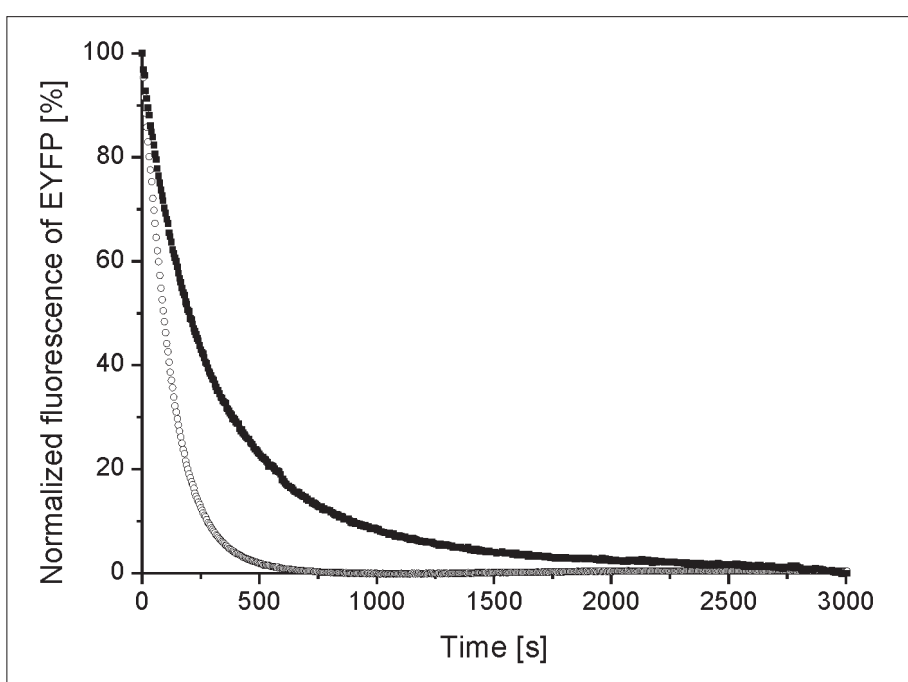

Fig. 2. Decay of eYFP fluorescence emission at $70^{\circ} \mathrm{C}$. Solutions of THS-eYFP-eCFP ( $\square)$ and $\operatorname{eYFP}(\mathrm{O})$.

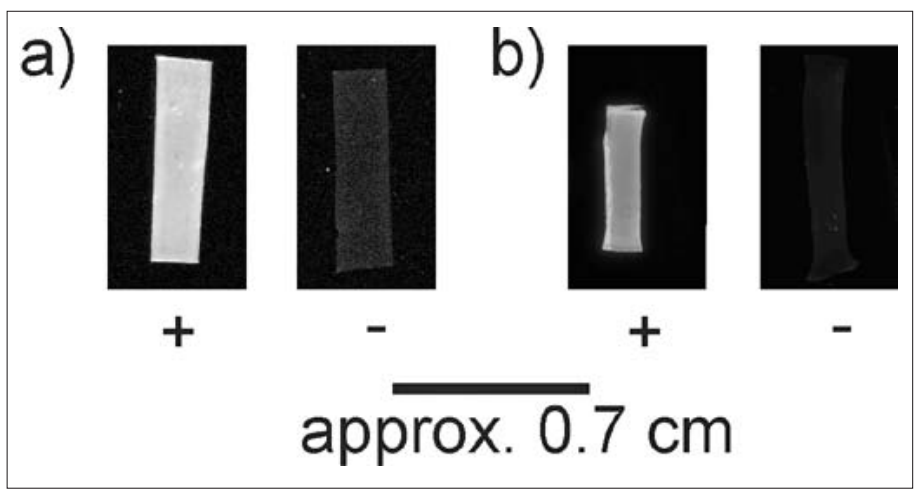

Fig. 3. Fluorescence scans of a) PEG and b) PAAm containing THS-eCFP-eYFP (+) and without proteins $(-)$

\section{Mechanical Deformation of Samples and Self-reporting of Damaged Areas}

The polyacrylamide samples were used as model systems to prove the concept of self-reporting materials based on protein mechanophores. To this end, dried samples were uniaxially strained until they tore. Fracturing is preceded by the formation of microcracks. These cracks widen and, eventually, one crack collapses and the sample snaps. The area surrounding the fracture face was then investigated by confocal microscopy, which allowed recording fluorescence images: intensity FRET and FLIM (Fig. 4). Clearly, variations in intensity FRET and mean fluorescence lifetime are co-localized with the microcracks seen in the fluorescence image. Both methods showed that FRET efficiency was higher, i.e. the distance between the fluorophores was shorter, within the vicinity of microcracks than in the bulk of the polymer. Control experiments with

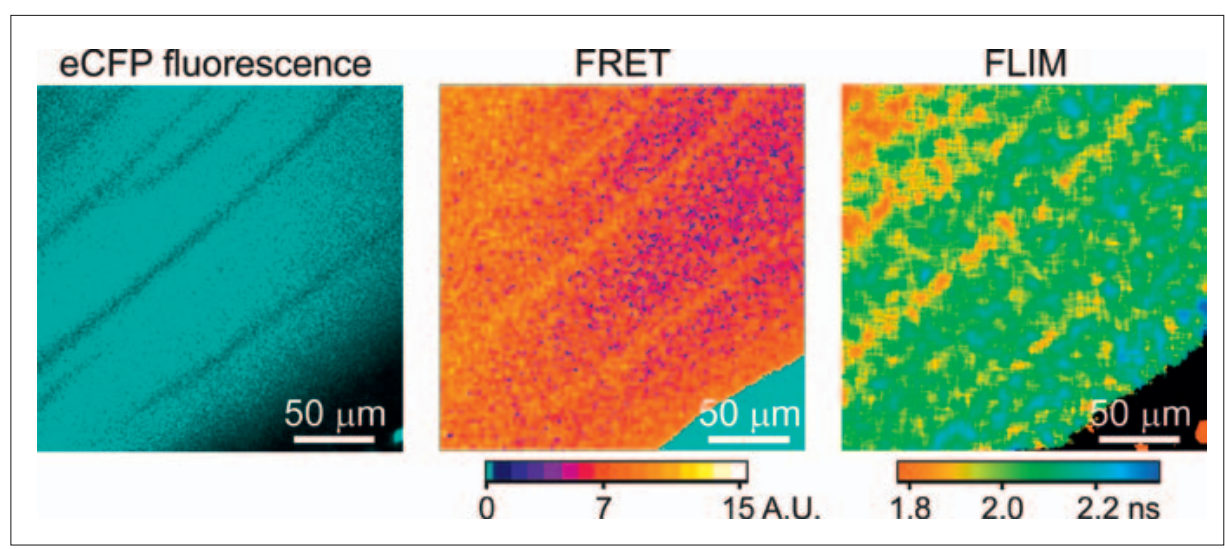

Fig. 4. Confocal fluorescence microscopy analysis of damaged PAAm-THS-eCFP-eYFP. The sample was uniaxially strained until it tore. The fluorescence emission in the blue channel upon excitation of eCFP, intensity-based FRET, and mean lifetime of the eCFP (calculated from FLIM) are shown. The fracture face is perpendicular to the plane of the image in the lower right hand corner of the micrographs. Reproduced with permission from ref. [26]. Copyright 2009 Wiley-VCH Verlag $\mathrm{GmbH} \& \mathrm{Co}$. KGaA. 


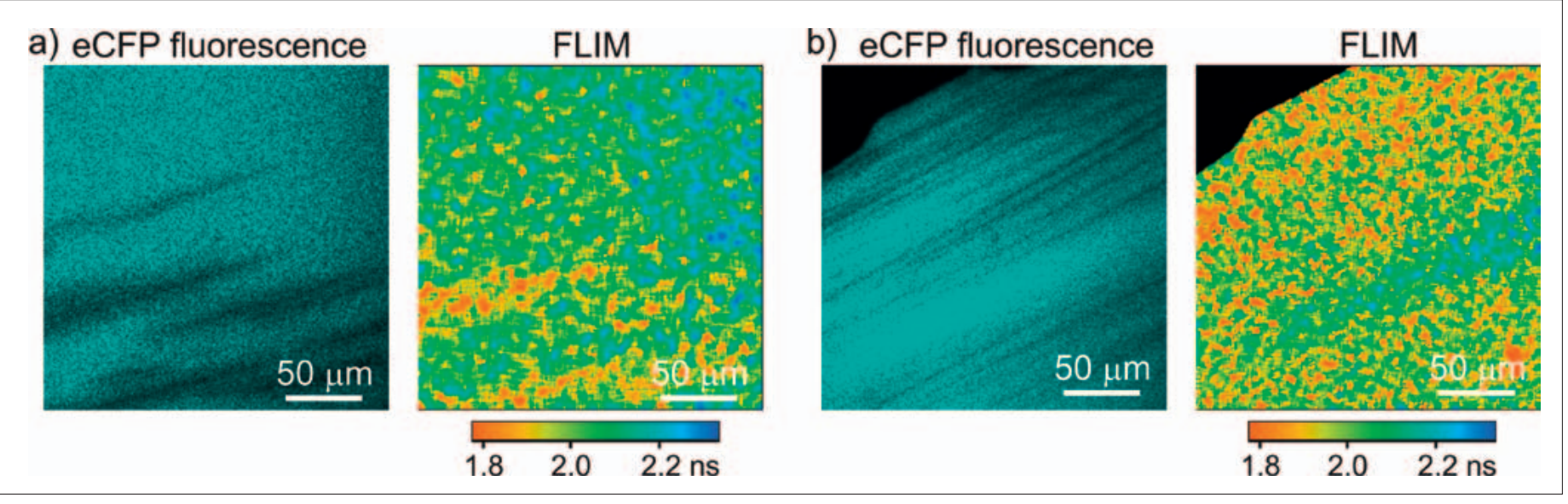

Fig. 5. Confocal fluorescence microscopy images of damaged PAAm-THS-eCFP-eYFP samples. FLIM shows the self-reporting effect in areas with microcracks. a) Region close to the micrograph presented in Fig. 4. b) Cracked area close to the fracture face of another sample. The fracture face is perpendicular to the plane of the image in the upper left hand corner of the micrographs.

poly(acrylamide) containing bare eCFP or THS encapsulating only eCFP did not show any differences in FLIM response between the bulk of the polymers and the vicinity of microcracks. [26]

Fig. 5a shows a FLIM of an adjacent region to the one previously published, where more of the non-damaged bulk of the sample is visible. Fig. $5 \mathrm{~b}$ depicts another typical sample in FLIM. Large areas rich in microcracks are observed, where the mean fluorescence lifetime of eCFP is low, indicating a higher FRET efficiency. Only a small ribbon of relatively undamaged bulk polymer in the middle of the micrograph was able to survive the stress. Here, the mean lifetime is higher; i.e. FRET efficiency is lower.
At first glance, these results are counterintuitive, because they indicate that the distance between the fluorophores decreases during crack formation. A compression effect can be ruled out because the fluorescent proteins are embedded in the THS, which represents a scaffold. Thus, the explanation is that some of the THS is strained in the bulk of the polymer and relaxes back to a more compact, possible native, conformation upon crack-formation (Fig. 6). An observation that supports this hypothesis is that the FRET efficiency in the non-damaged bulk of the polymer is rather low. Moreover, poly(acrylamide) gels and networks are known to possess an inhomogeneous distribution of crosslinking points, with highly crosslinked do-

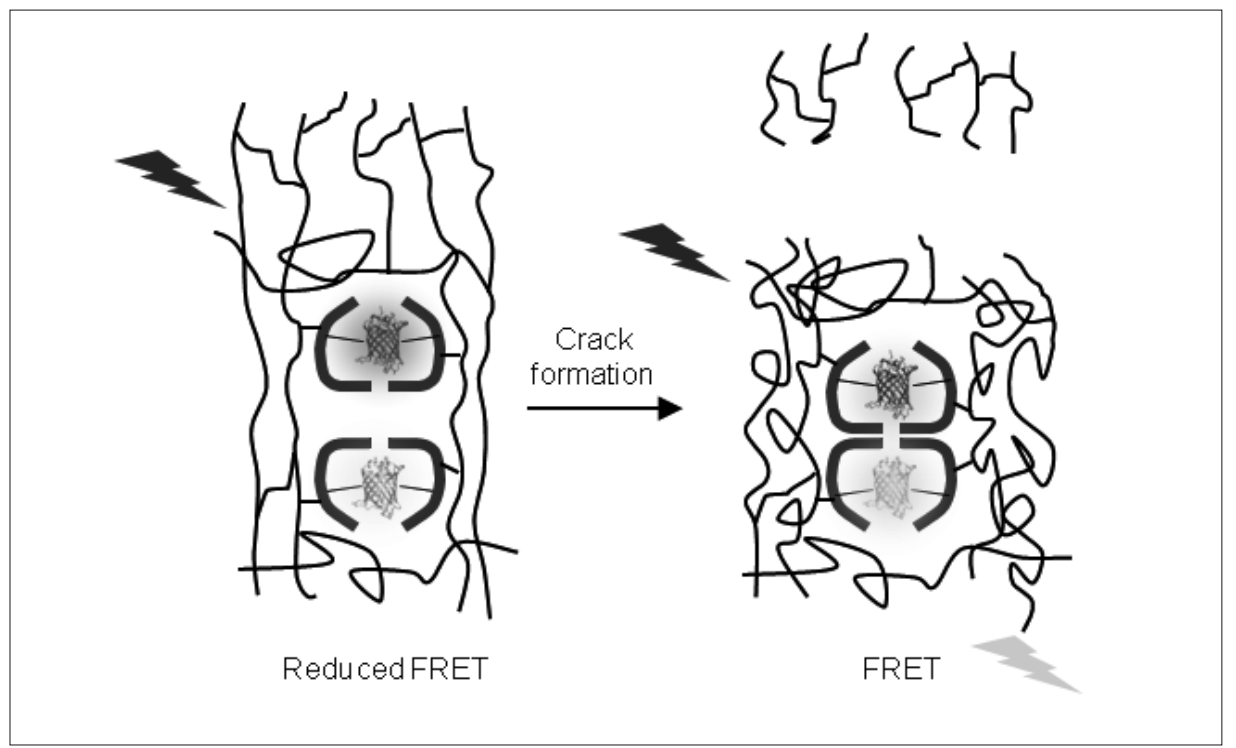

Fig. 6. Schematic depiction of self-reporting mechanism that reports the formation of cracks by a change in FRET efficiency. THS-eCFP-eYFP is covalently incorporated into a glassy polymeric network. Internal stress within this hybrid material leads to deformation of the THS native structure and cavity separation. Upon formation of cracks, the polymer and THS in the vicinity of a crack relaxes, which can be detected by increase in the emission intensity of the FRET acceptor eYFP, or by a shorter mean lifetime of the FRET donor eCFP. Reproduced with permission from ref. [26]. Copyright 2009 Wiley-VCH Verlag GmbH \& Co. KGaA.

mains embedded in a less densely crosslinked matrix. ${ }^{[29]}$ Thus, upon sample preparation, especially during the drying step, residual stress built up in the sample, e.g. due to different deswelling ratios of the domains. This stress transfers to the embedded protein-mechanophores. Crack formation is accompanied by a local relaxation of residual stress, presumably resulting in a relaxation of the THS as well.

\section{Conclusion}

Polymeric materials have been doped with functional proteins in order to generate hybrid materials that are able to report damages which are barely visible with blank eye. To the best of our knowledge the highlighted work was the first to proof that proteins can change properties in respond to a deformation of a polymer matrix, thus acting as mechanophores. A mechanophore system as complex as the one presented here is definitely interesting from a fundamental scientific point of view. It shows that the mechanical properties of protein scaffolds can be used to localize force-induced changes in a polymer. However, the THS-eCFP-eYFP mechanophore might be too complicated to ever find its way into real-world applications. Therefore, the group of N.B. is currently investigating bare fluorescent proteins as mechanophores for self-reporting polymeric materials. But this is a different story that will be revealed soon in future publications.

\section{Experimental}

Protocols for the expression of the proteins, their purification, modification and conjugation as well as the synthesis of PAAm-THS-eCFP-eYFP hybrid materials, and most analytical methods, have 
been previously published in the paper highlighted here.[26]

\section{Temperature Stability of Proteins}

Fluorescence measurements were carried out on a FluoroMax-4 spectrofluorometer (HORIBA Jobin-Yvon) equipped with a Peltier-thermostatted cuvette holder. Kinetics were obtained at $70{ }^{\circ} \mathrm{C}$ from liquid samples in a quartz sub-micro fluorimeter cell (Type 16, nominal volume $50 \mu \mathrm{l}$, Starna Scientific). Single wavelength emission kinetics were recorded $\left(\lambda_{\mathrm{Ex}}=490 \mathrm{~nm}\right.$, slit width $3 \mathrm{~nm}, \lambda_{\mathrm{Em}}=527 \mathrm{~nm}$, slit width $3 \mathrm{~nm}$, integration time $1 \mathrm{~s}$ ). The following solutions were analyzed: THS-eCFP-eYFP (0.009 $\mathrm{mg}$ eYFP $\left.\mathrm{ml}^{-1}\right)$, and eYFP modified with SANH $\left(0.018 \mathrm{mg}^{\mathrm{eYFP}} \mathrm{ml}^{-1}\right)$ in $20 \mathrm{mM}$ Tris/HCl (pH 7.5, $1 \mathrm{mM}$ EDTA, $0.02 \% \mathrm{w} / \mathrm{v} \mathrm{NaN}_{3}$ ).

\section{Fluorescence Scans}

Polymers were scanned on a Typhoon 9410 gel imager (Amersham Biosciences) in fluorescence mode in order to detect eYFP fluorescence.

\section{Synthesis of PEG-THS-eCFP-eYFP Materials}

$\alpha, \omega$-Acrylate-terminated PEG (AAPEG-AA) was synthesized by dissolving $\alpha, \omega$-hydroxy-terminated PEG (20 g; 8000 $\mathrm{g} \mathrm{mol}^{-1}$, EMD Chemicals) and freshly distilled acryloyl chloride $(0.809 \mathrm{ml}, 0.905 \mathrm{~g}$, $10.0 \mathrm{mmol}$ ) in $250 \mathrm{ml}$ anhydrous THF at $50{ }^{\circ} \mathrm{C}$. After stirring for $2 \mathrm{~h}$ at this temperature, triethyl amine $(1.39 \mathrm{ml}, 1.01 \mathrm{~g}$, $10.0 \mathrm{mmol}$ ) was added. The mixture was stirring at $50{ }^{\circ} \mathrm{C}$ for an additional $2 \mathrm{~h}$. The formed precipitate was removed by filtration and the solution concentrated in vacuum. The residue was redissolved in water and the $\mathrm{pH}$ was adjusted to 6.0 by addition of diluted $\mathrm{NaOH}$. Then the polymer was extracted into dichloromethane, and the combined organic phases were dried over $\mathrm{MgSO}_{4}$. Finally, the polymer was purified by precipitation from dichloromethane by addition of diethylether.

To synthesize PEG-THS-eCFP-eYFP hybrid materials, AA-PEG-AA (20 mg) was dissolved in $40 \mu \mathrm{l}$ of $0.5 \mathrm{mg} \mathrm{ml}^{-1}$ THSeCFP-eYFP solution in $50 \mathrm{mM}$ sodium phosphate buffer ( $\mathrm{pH} 7.4,75 \mathrm{mM} \mathrm{NaCl})$.

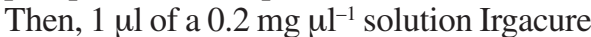
2959 (Ciba, now part of BASF) in DMSO was added. The resulting solution was mixed and poured into a $10 \times 10 \times 0.5 \mathrm{~mm}^{3}$ mold consisting of a PDMS gasket glued to a glass slide. A second glass slide was placed on top, sealing the mold. Polymerization was carried out by irradiating for $3 \mathrm{~min}$ with a longwave UV lamp (Black Ray B100 AP) at room temperature. The polymer gel was removed from the mold and incubated overnight in $5 \mathrm{ml}$ of $100 \mathrm{mM}$ sodium phosphate buffer ( $\mathrm{pH} 7.4,150 \mathrm{mM} \mathrm{NaCl}$ ) while shaking at $4{ }^{\circ} \mathrm{C}$ in order to equilibrate the $\mathrm{pH}$ and to extract any unreacted reagents or non-crosslinked polymer chains. Next, the polymer was transferred into $5 \mathrm{ml}$ water (adjusted to $\mathrm{pH} 7.4$ with $\mathrm{NaOH}$ ) and incubated for $5 \mathrm{~h}$ while shaking at $4{ }^{\circ} \mathrm{C}$. The gel was cut into $3 \mathrm{~mm}$ wide strips and air-dried for $40 \mathrm{~h}$ at room temperature.

\section{Acknowledgements}

This work was funded by the Air Force Office of Scientific Research (FA9550-07-10116). N.B. gratefully acknowledges a Marie Curie Intra European Fellowship.

Received: January 21, 2011

[1] M. M. Caruso, D. A. Davis, Q. Shen, S. A Odom, N. R. Sottos, S. R. White, J. S. Moore, Chem. Rev. 2009, 109, 5755.

[2] D. Y. Wu, S. Meure, D. Solomon, Prog. Polym. Sci. 2008, 33, 479.

[3] S. D. Bergman, F. Wudl, J. Mater. Chem. 2008, $18,41$.

[4] R. P. Wool, Soft Matter 2008, 4, 400.

[5] S. R. White, N. R. Sottos, P. H. Geubelle, J. S. Moore, M. R. Kessler, S. R. Sriram, E. N. Brown, S. Viswanathan, Nature 2001, 409, 794

[6] M. Motuku, U. K. Vaidya, G. M. Janowski, Smart Mater. Struct. 1999, 8, 623.

[7] J. W. C. Pang, I. P. Bond, Composites: Part A. 2005, 36, 183 .

[8] J. W. C. Pang, I. P. Bond, Compos. Sci. Technol. 2005, 65, 1791

[9] S. M. Bleay, C. B. Loader, V. J. Hawyes, L. Humberstone, P. T. Curtis, Composites: Part A 2001, 32, 1767

[10] M. S. Mueller, L. Hoffmann, A. Sandmair, A. W. Koch, IEEE J. Quantum Electron. 2009, 45, 547.
[11] D. A. Davis, A. Hamilton, J. Yang, L. D. Cremar D. Van Gough, S. L. Potisek, M. T. Ong, P. V. Braun, T. J. Martinez, S. R. White, J. S. Moore, N. R. Sottos, Nature 2009, 459, 68 .

[12] C. K. Lee, D. A. Davis, S. R. White, J. S. Moore, N. R. Sottos, P. V. Braun, J. Am. Chem. Soc. 2010, 132, 16107

[13] C.-M. Chung, Y.-S. Roh, S.-Y. Cho, J.-G. Kim, Chem. Mater. 2004, 16, 3982.

[14] K. Anuchai, C. Weder, R. Magaraphan, Plast. Rubber Compos. 2008, 37, 281.

[15] B. R. Crenshaw, M. Burnworth, D. Khariwala, A. Hiltner, P. T. Mather, R. Simha, C. Weder, Macromolecules 2007, 40, 2400.

[16] J. Kunzelman, B. R. Crenshaw, M. Kinami, C. Weder, Macromol. Rapid Commun. 2006, 27, 1981.

[17] B. R. Crenshaw, C. Weder, Macromolecules 2006, 39, 9581.

[18] M. Kinami, B. R. Crenshaw, C. Weder, Chem Mater. 2006, 18, 946.

[19] B. R. Crenshaw, C. Weder, Chem. Mater. 2003 $15,4717$.

[20] H. Dietz, M. Rief, Proc. Natl. Acad. Sci. USA 2004, 101, 16192.

[21] M. Bertz, A. Kunfermann, M. Rief, Angew. Chem., Int. Ed. 2008, 47, 8192

[22] L. Ditzel, J. Löwe, D. Stock, K.-O. Stetter, H. Huber, R. Huber, S. Steinbacher, Cell 1998, 93, 125 .

[23] M. G. Bigotti, A. R. Clarke, Arch. Biochem Biophys. 2008, 474, 331

[24] F. Valle, J. A. DeRose, G. Dietler, M. Kawe, A Plückthun, G. Semeza, Ultramicroscopy 2002, 93,83

[25] M. A. Rizzo, G. Springer, K. Segawa, W. R. Zipfel, D. W. Piston, Microsc. Microanal. 2006, $12,238$.

[26] N. Bruns, K. Pustelny, L. M. Bergeron, T. A. Whitehead, D. S. Clark, Angew. Chem., Int. Ed. 2009, 48, 5666 .

[27] C. R. Booth, A. S. Meyer, Y. Cong, M. Topf, A. Sali, S. J. Ludtke, W. Chiu, J. Frydman, Nat. Struct. Mol. Biol. 2008, 15, 746.

[28] S. H. Bokman, W. W. Ward, Biochem. Biophys. Res. Commun. 1981, 101, 1372.

[29] A. M. Hecht, R. Duplessix, E. Geissler, Macromolecules 1985, 18, 2167. 